\title{
Modelando una propuesta conceptual para analizar características psicosociales de emprendedores sociales peruanos
}

\section{Modeling a conceptual proposal to analyze psychosocial characteristics of Peruvian social entrepreneurs}

\author{
Angela Vera Ruiz ${ }^{\mathrm{a},}$, María Ángela Prialé Valle ${ }^{\mathrm{b}}$, Agustín Espinosa Pezziaa, \\ Erle Frances Ninahuanca López

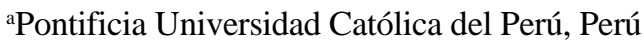 \\ bUniversidad del Pacífico, Perú
}

\begin{abstract}
Resumen
Objetivo: este estudio tiene como objetivo proponer un abordaje comprensivo de cinco dimensiones teóricas características de los emprendedores sociales, para plantear un modelo conceptual que las analiza desde los constructos psicosociales macro de Creencias y Prácticas. Método: se desarrolló un estudio cualitativo, con 16 emprendedores sociales de la ciudad de Lima (Perú), cuyas trayectorias, por medio de un análisis temático deductivo, se analizaron y operativizaron en tanto creencias y/o prácticas en variables psicosociales más específicas como valores, actitudes, conductas y rasgos. Resultados: la dimensión de Aprecio por Prácticas Sostenibles (APS), de carácter actitudinal, resulta ser el eje articulador en el que confluyen las experiencias de todos los participantes para concretar su visión social, de carácter axiológico, promoviendo conductas competentes de innovación, redes y retornos financieros. Conclusión: se discuten los resultados enfatizando la necesidad de profundizar en el estudio de la dimensión APS de manera contextualizada a las problemáticas sociales del Perú.
\end{abstract}

Palabras clave: emprendedor social; dimensiones del emprendimiento social; creencias; prácticas.

Para citar este artículo:

Vera, A., Prialé, M. A., Espinosa, A., \& Ninahuanca, E. F. (2020). Modelando una propuesta conceptual para analizar características psicosociales de emprendedores sociales peruanos. Liberabit, 26(1), e256. https://doi.org/10.24265/liberabit.2020. v26n1.03

*averar@pucp.pe

\begin{abstract}
Objective: this study aims to propose a comprehensive approach to five theoretical dimensions, characteristic of social entrepreneurs, to design a conceptual model that analyzes them based on macro-level psychosocial constructs such as beliefs and practices. Method: a qualitative study was conducted with 16 social entrepreneurs from the city of Lima (Peru). Using a deductive thematic analysis, their careers were examined and made operative based on their beliefs and/or practices, and on more specific psychosocial variables such as values, attitudes, behaviors and traits. Results: the attitudinal dimension of Positive Attitudes Towards Sustainable Practices (PASP) is the articulating main point in which all participants' experiences come together to define their social vision, of axiological nature, promoting competent behaviors including innovation, networks and financial returns. Conclusion: the results highlight the need to deepen the study of the PASP dimension focused on the Peruvian social issues.
\end{abstract}

Keywords: social entrepreneur; social entrepreneurship dimensions; beliefs; practices.

Este es un artículo Open Access bajo la licencia Creative Commons Atribución-NoComercial-CompartirIgual 4.0

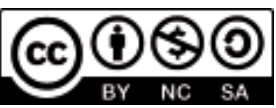

Universidad de San Martín de Porres, Lima - Perú http://ojs3.revistaliberabit.com 


\section{Introducción}

Durante el siglo XXI, las denominadas empresas sociales se han posicionado como organizaciones híbridas que, recurriendo a estrategias de mercado, buscan resolver problemáticas sociales o ambientales que nadie más está resolviendo (Abhi, 2017; Chell, Spence, Perrini, \& Harris, 2016; Mair \& Martí, 2006). El objetivo del emprendimiento social es generar impactos socioambientales positivos empleando modelos operativa y financieramente viables (Defourny \& Nyssens, 2017; Ojeda \& Rodríguez, 2015). El emprendimiento social se diferencia del emprendimiento comercial, en tanto el segundo, si bien es socialmente valioso, prioriza la rentabilidad financiera como forma principal de medición del valor creado (Young, 2006).

En la literatura, se destaca al emprendedor como aquel individuo que cumple el rol catalizador para la puesta en marcha del emprendimiento, por lo cual resulta relevante su estudio y caracterización (Pollack, Rutherford, Seers, Coy, \& Hanson, 2016; Smith, Smith, \& Shaw, 2017). Diversas investigaciones han trabajado prolíficamente las características psicosociales del emprendedor comercial y los antecedentes que le llevan a crear y mantener su proyecto (Brandstätter, 2011; Luca, 2017; Zhao \& Seibert, 2006). En el caso del emprendedor social (ES), dado que su estudio es más reciente, el desarrollo teórico se encuentra aún disgregado en aportes de distintas disciplinas (Curry, Donker, \& Michel, 2016; Estrin, Mickiewicz, \& Stephan, 2016; Ovais \& Li, 2016; Waddock \& Steckler, 2016).

Si bien en la caracterización del ES se destacan atributos como la innovación para transformar la sociedad, lo cual crea unidad de propósito o propensión a asumir riesgos con proactividad (Humbert \& Roomi, 2018), son reducidos los trabajos que proponen un modelo o tipología conceptual general (Nga \& Shamuganathan, 2010; Ojeda \& Rodríguez, 2015) para entender las especificidades psicosociales del ES en un contexto socioeconómico determinado (Sengupta, Sahay, \& Croce, 2017; Zozimo, Jack, \& Hamilton, 2017).

En consecuencia, existe un conjunto de conceptos sobre el ES que, aunque estén semánticamente relacionados, aún no son suficientemente teorizados desde una mirada psicosocial que permita explicar cómo y por qué el ES es diferente a sus homólogos comerciales en un ecosistema emprendedor específico dentro del cual crean valor social (El Ebrashi, 2013; Vera et al., 2016). El presente estudio identifica dos vacíos: el primero, da cuenta de la necesidad de proponer un modelo conceptual integrador de las características psicosociales de los ES; el segundo, implica reconocer que dicho modelo debe analizarse en el contexto especifico del ES, pues, aun en los casos donde se estudian experiencias y características del ES, los análisis suelen omitir las condiciones del contexto socioeconómico que lo rodea, restringiendo la comprensión situada de su comportamiento (Bikse, Rivza, \& Riemere, 2015; Sastre-Castillo, Periz-Ortiz, \& Danvila-Del Valle, 2015).

El presente estudio pretende aportar en la reducción de los dos vacíos identificados, proponiendo, desde una perspectiva psicosocial, un modelo conceptual integrador para analizar las experiencias de emprendedores sociales de Lima, a la luz de sus trayectorias y de las particularidades del contexto local, para reconocer sus atributos emprendedores distintivos.

El documento presenta, en primer lugar, una revisión conceptual en la línea de la propuesta de cinco dimensiones del ES planteada por Nga y Shamuganathan (2010). Seguidamente, se plantean constructos teóricos psicosociales en el marco teórico de prácticas y creencias para analizar las dimensiones del ES revisadas. El marco teórico se cierra con una contextualización del emprendimiento social en el Perú. Posteriormente, se explica el método de investigación cualitativa del análisis temático-deductivo, elegido para abordar en profundidad un tema poco explorado localmente (Braun \& Clarke, 2006; Creswell, 2013). Finalmente, 
se reportan y discuten los hallazgos desde los temas comunes a todos los ES participantes y, a partir de ellos, se conceptualizan y organizan las dimensiones del ES en una propuesta de modelo conceptual psicosocial situado.

\section{Las dimensiones teóricas del emprendedor social (ES)}

La literatura señala que el ES difiere en sus objetivos del emprendedor comercial, ya que busca resolver fallas de mercado producidas por la inequidad distributiva, la inadecuada gestión de bienes públicos y el escaso control de externalidades (El Ebrashi, 2013). El ES demuestra una visión más integral sobre el impacto social de su emprendimiento y una mayor empatía, juicio moral, autosuficiencia y capacidad de cooperación con las personas con las que se relaciona (Austin, Stevenson, \& Wei-Skiller, 2006; Mair \& Martí, 2006). Los distintos atributos personales identificados en los ES son recogidos por Nga y Shamuganathan (2010), tras una exhaustiva revisión de la literatura, en una propuesta de cinco dimensiones: (1) Visión social, (2) Aprecio por prácticas sostenibles, (3) Capacidad innovadora, (4) Habilidad para desarrollar redes de contacto y (5) Habilidad para generar retornos financieros, las que a continuación se detallan.

Visión social: definida como el sentido de responsabilidad, compromiso, pasión y conexión emocional que tiene el ES con una causa ambiental o social desatendida (Abhi, 2017; Ovais \& Li, 2016). La visión social se considera la condición previa para la creación de valor social, siendo una característica clave del ES (Choi \& Majumdar, 2014; Írengün \& Arikboga, 2015; Mair \& Martí, 2006). Algunos autores señalan que se relaciona con vivencias primarias de carácter traumático (como la muerte de un ser querido) o transformadoras (como viajes o trabajo comunitario) (Ovais \& Li, 2016; Waddock \& Steckler, 2016).

Aprecio por prácticas sostenibles: ilustra el interés del ES por emplear prácticas y modelos económicos innovadores (Zhang \& Swanson, 2014) que aseguren la permanencia de la organización en el tiempo y generen un balance de intereses económicos, sociales y ambientales, o «triple resultado» (Graddy-Reed, 2018; Kuckertz \& Wagner, 2010; Savitz \& Weber, 2006). El aprecio por prácticas sostenibles nace de la motivación por solucionar problemáticas socioambientales (Humbert \& Roomi, 2018; Whitman, 2011) y de un estado de conciencia crítica, social y moral que lleva al ES a considerar que las organizaciones e individuos son parte de un ecosistema interconectado (Peredo, Haugh, \& McLean, 2017; Savitz \& Weber, 2006).

\section{Habilidad para desarrollar redes de contacto:} se refiere a la capacidad para mantener relaciones positivas, formales o informales con personas del entorno (Ovais \& Li, 2016) para acceder a recursos, capital financiero, clientes potenciales e información (Pollack et al., 2016) y expandir los alcances del emprendimiento (Bikse, Rivza, \& Riemere, 2015; Sesen, 2013). Son claves la reputación, la confianza y la credibilidad que el emprendedor social cultiva entre sus contactos, dada la calidad y permanencia de sus relaciones a lo largo de su trayectoria (Abhi, 2017; Carter, Collins, \& Beal, 2017; Gurrieri, 2013). Las redes reducen la incertidumbre, impulsan el espíritu emprendedor y previenen conflictos (Carter et al., 2017; Kristiansen \& Ryen, 2002).

Capacidad innovadora: es una característica fundamental e inherente del ES (Bikse et al., 2015; Weerawardena \& Sullivan, 2006) que lo conduce a transformar cualquier factor clave de su modelo de operación para generar una plataforma de recursos (Mair \& Martí, 2006; Pless, 2012) en sus procesos, tecnologías y soluciones colectivas (Carter et al., 2017), con lo cual logra resolver problemas sociales crónicos de manera efectiva (Rahdari, Sepasi, \& Moradi 2016). La capacidad innovadora se desarrolla y nutre en redes de intercambio de capital intelectual y social y en el empoderamiento de mercados desatendidos (Pralahad, 2010; Sastre-Castillo et al., 2015). 
Generación de retornos financieros: da cuenta de aprovechar oportunidades y competir por recursos económicos que garanticen el funcionamiento a largo plazo del emprendimiento, el cumplimiento de sus objetivos sociales y su escalamiento (Írengün \& Arikboga, 2015; Mair \& Martí, 2006). Esta dimensión conduce al ES a elaborar planes de negocios (Curry et al., 2016; Rahdari et al., 2016), a innovar modelos empresariales existentes y a recurrir a fondos públicos, consultores e inversionistas que aporten recursos cruciales por lo menos en las fases iniciales del emprendimiento (Graddy-Reed, 2018; MendozaAbarca \& Mellema, 2016).

\section{Constructos psicosociales para proponer un modelo conceptual de las dimensiones del ES}

Si bien el esfuerzo conceptual de Nga y Shamuganathan (2010) constituye un referente para el campo de estudio, la literatura revisada no aclara cuál es la naturaleza psicosocial de cada una de las dimensiones; no define si se trata de rasgos o actitudes o conductas, entre otras posibles variables psicológicas distintivas del ES. Se proponen, entonces, dos constructos psicosociales macro: las creencias y las prácticas, dentro de los cuales se pueden definir variables más específicas que aportan al estudio de las dimensiones del ES.

Creencias: las creencias son teorías o explicaciones fundadas en los sentimientos y experiencias personales que elabora cada individuo ante la ausencia de un conocimiento específico para poder adaptarse a su entorno, interpretarlo y guiar su propio comportamiento (Serrano, 2010; Rodrigo, Rodríguez, \& Marrero, 1993). Son implícitas o no conscientes, se generan en el intercambio con el entorno y permiten la síntesis de una visión del mundo, variando de acuerdo al contexto espacial y temporal del sujeto, por lo que suelen ser asumidas como universales y verdaderas (Rodrigo et al., 1993). Dos variables psicosociales asociadas a las creencias y que se proponen para estudiar al ES son las siguientes: (1) valores, los cuales se definen como creencias centrales meta-motivacionales referidas a modos de conducta deseables que orientan la evaluación y selección de comportamientos, personas o eventos, transcendiendo situaciones específicas (Schwartz, 1992, 2010) y (2) actitudes, las cuales son entendidas como una organización relativamente estable de creencias evaluativas, sentimientos y tendencias de conducta que se asocian con, o están dirigidas hacia, una entidad particular u objeto (Eagly \& Chaiken, 2005; Morris \& Maisto, 2011).

Prácticas: se trata de formas rutinarias de conducta que se componen por actividades corporales, mentales, significados y saberes prácticos, emociones y motivaciones, todos interconectados de maneras específicas según el tipo de actividad, las cuales se materializan en una relación directa y cotidiana del sujeto con el mundo (Reckwitz, 2002). Son formas de hacer o decir algo, que surgen en la interrelación espacio-temporal de las personas y que dan cuenta de sus competencias, afectos y recursos, siendo la coexistencia activa de estos tres elementos lo que permite ejecutar la práctica en sí (Shove, Pantzar, \& Watson, 2012). Dos variables específicas asociadas a este constructo son las siguientes: (1) rasgos, entendidos como el conjunto de cualidades psicológicas individuales y estructurales que influyen en los patrones característicos de conducta de un individuo en distintas situaciones a lo largo del tiempo (Zhao \& Seibert, 2006) y (2) competencias, siendo el eslabón observable producto de complejos estados internos (Freixa, 2003) que expone los saberes y habilidades para la ejecución de una práctica, configurando elementos conductuales en forma de destrezas (Shove et al., 2012).

Como se presentará en el análisis de resultados, proponer estos dos constructos macro con variables psicosociales más específica, da la posibilidad de identificar y analizar procesos cognitivos, afectivos y conductuales asociados a la experiencia de los ES, enriquecida por la particularidad del contexto. Razón por la cual resulta relevante presentar, a continuación, el caso específico peruano. 


\section{Contexto para el emprendimiento social en el Perú}

El Perú es reconocido como una de las economías más estables de Latinoamérica y el Caribe, además de figurar como uno de los países de mayor iniciativa emprendedora en el mundo, siendo el emprendimiento altamente valorado como carrera profesional por parte del 82.4\% de los peruanos (Banco Mundial, 2016; Global Entrepreneurship Monitor [GEM], 2018). Con respecto al emprendimiento social en el Perú, su práctica tiene una historia relacionada con tradiciones como la economía solidaria, el tercer sector y el cooperativismo, así como con figuras organizacionales más recientes como las microfinancieras. En la última década, los emprendimientos sociales han despertado el interés de la academia, los formuladores de políticas públicas y el público en general, ganando visibilidad en el país (Caballero, Fuchs, Prialé, \& Nga, 2014; Farber, Caballero, Prialé, \& Fuchs, 2015; Vera et al., 2016).

En relación a investigaciones empíricas realizadas en Perú con ES, Caballero et al. (2014) llevaron a cabo un estudio cuantitativo con ES peruanos en el que correlacionaron las cinco dimensiones de Nga y Shamuganathan (2010) con el modelo de rasgos de personalidad Big Five. De esta manera encontraron que las dimensiones de Visión social y Aprecio por prácticas sostenibles son explicadas por los rasgos de personalidad, rigurosidad y agradabilidad, denotando este último un modo distinto de ser emprendedor (Írengün \& Arikboga, 2015; Ovais \& Li, 2016; Zhao \& Seibert, 2006). Otros estudios recientes, que analizan las características y condiciones de formación y sostenibilidad de emprendimientos sociales en el país, señalan que la mayoría de estas iniciativas desarrollan labores educativas y de servicios, y que el ecosistema emprendedor social peruano se encuentra centralizado en la capital requiriendo aún un mayor impulso y búsqueda de sostenibilidad en el tiempo para que estas iniciativas se extiendan en todo el país (Giraldo-Mejía \& VaraHorna, 2018; Kunan \& Universidad Científica del Sur, 2019; Vera et al., 2016).
La literatura local explica que la duración de los emprendimientos en el Perú, en general, es relativamente corta dada la informalidad y el bajo soporte institucional y estatal (Ghezzi \& Gallardo, 2013; Latinobarómetro, 2017), quedando desatendidas necesidades de la población y el ambiente, que finalmente amplían las brechas sociales a todo nivel (Caballero et al., 2014; Consejo Nacional de Ciencia y Tecnología [CONCYTEC], 2016). Por esta razón, ampliar el estudio del emprendimiento y, en particular, el de las características de los ES, favorecería la promoción de impactos positivos en una realidad local inequitativa y excluyente como la peruana, aportando al conocimiento psicológico aplicado al ámbito empresarial.

Es así que el objetivo del presente estudio es proponer un modelo conceptual integrador, desde una perspectiva psicosocial, para analizar las experiencias de emprendedores sociales de Lima. Se busca entender, desde las trayectorias de los participantes en el contexto local peruano, las condiciones en que se desarrollan algunos de sus atributos emprendedores distintivos.

\section{Método}

\section{Participantes}

El estudio contó con 16 participantes, 8 mujeres y 8 hombres, fundadores de emprendimientos sociales en Lima. La selección se realizó por un muestreo intencional, siendo los criterios de inclusión: el autoreconocimiento como emprendedor social y el aval de alguna institución reconocida en el ecosistema emprendedor social local (ONG, Incubadora, Centro de Emprendimiento, Patronato). Las edades de los participantes oscilan entre los 23 y 75 años de edad $(M=37)$. La antigüedad de sus empresas sociales varía desde 2 hasta 30 años $(M=8.5)$. Todos los emprendimientos fundados por los participantes pertenecen al sector de servicios. Las problemáticas generales que atienden incluyen: acceso a crédito, educación ciudadana, cuidado del medio ambiente, comercio justo, desarrollo del agro, inclusión laboral, 
ecoturismo y prestación de servicios de saneamiento y manejo de residuos. A todos los participantes se les presentó un consentimiento informado, el cual aceptaron de manera voluntaria, concediendo también la grabación de las entrevistas en medios de registro de voz. Para garantizar la confidencialidad, los nombres de los participantes y las empresas han sido omitidos en la presentación de los resultados.

\section{Instrumento}

Se construyó una guía semiestructurada de entrevista en profundidad, teniendo como referentes las cinco dimensiones de emprendimiento social presentadas en el marco teórico. La entrevista indagaba por las prácticas y las creencias en la historia de vida de la persona, asociadas a cada dimensión, profundizando en la reflexión sobre las condiciones personales que le llevaron a desarrollar su emprendimiento y reconocerse a sí mismo como ES. El instrumento fue validado por jueces y ajustado a nivel de lenguaje y fluidez tras la aplicación de un piloto.

\section{Procedimiento}

Los participantes fueron contactados a través de instituciones reconocidas que los avalan en el ecosistema emprendedor social local (ONG, Centros de Emprendimiento y Patronatos), haciéndoles la invitación a participar de manera voluntaria en el estudio. Las entrevistas se programaron de común acuerdo con los entrevistados y se llevaron a cabo en sus respectivos lugares de trabajo tras la firma del consentimiento informado, el cual explicitaba los términos de confidencialidad, participación libre y voluntaria, así como los objetivos de la investigación, la metodología y los términos de devolución del estudio. Las entrevistas tuvieron una duración promedio de 60 minutos y fueron transcritas para su posterior análisis.

\section{Análisis de datos}

Todas las entrevistas fueron analizadas utilizando el programa ATLAS.ti 7.5 y, empleando la metodología del análisis temático-deductivo (Braun \& Clarke, 2006), partió de las cinco dimensiones teóricas del emprendimiento social (Nga \& Shamuganathan, 2010) como categorías teóricas previamente establecidas y de los conceptos psicosociales de creencias y prácticas. El análisis se realizó en función de la información común brindada por todos los participantes, el cual se contrastó con el marco conceptual (Willig, 2013) para establecer los puntos en común entre las cinco dimensiones del ES y las variables psicosociales con las que están relacionadas (Creswell, 2013; Willig, 2013).

\section{Resultados y discusión}

La Tabla 1 resume los contenidos brindados por los ES respecto a las cinco dimensiones teóricas del emprendimiento social. Para cada dimensión se ha diferenciado aquella información que da cuenta de las creencias con las que los participantes asignan significado a cada dimensión y las prácticas con las que ellos ejecutan o materializan dichas creencias. A continuación, se reportan y discuten en detalle los análisis de cada dimensión.

\section{Visión social}

Desde las experiencias de los emprendedores, la visión social se configura como un elemento misional, emocional y axiológico que les motiva a llevar a cabo el emprendimiento para luchar por una causa que se considera justa (Abhi, 2017; Chell et al., 2016; Írengün \& Arikboga, 2015; Popoviciu \& Popoviciu, 2011), explicitando la problemática concreta a solucionar.

Nosotros estamos naciendo como un agente de cambio... No es posible que un ser humano como tuì o como yo, tenga que buscar su comida en la basura, no puede ser. Tenemos que cambiar eso (Participante 1). 


\section{Tabla 1}

Resumen de resultados: contenidos comunes a nivel de creencias y prácticas para cada dimensión de emprendimiento social

\begin{tabular}{llc}
\hline Categorías & Creencia & Práctica \\
\hline
\end{tabular}

Visión Social

Sentirse comprometido y emocionado con la misión de hacer el bien y luchar por una causa que se considera justa y trascendente y que orienta, de manera eudaimónica, hacia la autorrealización.

Aprecio por

prácticas

sostenibles

Conciencia de interdependencia con un contexto donde hay desequilibrios en el que se asume una posición personal de responsabilidad.

Capacidad

Innovadora

Habilidad para desarrollar redes de contacto

Habilidad para generar retornos financieros
Valoración positiva de la incertidumbre para flexibilizarse y descentrarse de las propias necesidades.

Se conciben las redes y relaciones como construcciones colectivas que hacen posible que exista el emprendimiento y dan sentido al logro de la meta común.

Se concibe como una condición necesaria, pero difícil de lograr.Representa dificultades y sacrificios y es la dimensión menos estructurada en sus iniciativas.
Experimentar estados emotivos, positivos, satisfactorios y optimistas, en coherencia con los valores personales, declarando una misión clara para la iniciativa emprendedora.

Experiencias de vida como ejemplos para concretar las ideas en hechos. Equilibrar lo afectivo y racional de manera ética y empática para planificar actividades en favor de la sostenibilidad.

Habilidad para identificar necesidades y oportunidades de acción. Flexibilidad y adaptación frente a situaciones diversas.

Integración y participación en redes y eventos, usando tecnologías, «boca a boca», medios de comunicación y redes sociales para visibilizarse local e internacionalmente.

Se llevan a cabo actividades multitarea, de manera empírica y recursiva para optimizar costos. Se toman medidas razonables de austeridad económica.

Nota: elaboración propia.

Todos los emprendedores participantes se remiten a las razones sociales por las que operan y persisten en el tiempo sus emprendimientos, manifestando un sentido de bienestar y autorrealización en el logro de sus objetivos.

Tú tienes que hacer lo que te apasiona. Yo encontré mi pasión en el emprendimiento social... Si tuì haces lo que realmente te llena como persona, puedes hacer cambios transformacionales porque te sientes bien, te sientes satisfecho de estar haciendo ese cambio, entonces vas a hacerlo por mucho tiempo (Participante 4).

La visión social permite llevar a cabo la misión del emprendimiento, en tanto conecta emocionalmente al ES con una lucha que afronta de forma optimista y que considera satisfactoria para declarar y planificar la realización de metas (Bacq \& Alt, 2018; Ovais $\& \mathrm{Li}, 2016)$. Esto conduce a un compromiso a largo plazo apoyado en la convicción de estar haciendo lo correcto con la ejecución de sus proyectos. Este hecho daría cuenta de un posicionamiento axiológico, relacionado a valores universalistas de cuidado y desarrollo del bienestar de otros, más allá de la satisfacción de intereses personales (Schwartz, 2010; García-Villaverde \& Valencia, 2009).

Yo creo que la principal motivación es un tema de los compromisos que uno va adquiriendo cuando empieza a transmitir lo que realmente quiere con su filosofía... estar sirviendo a la medida de los demás, te genera compromisos (Participante 3).

En los participantes, la visión social se asocia con una idea optimista del mundo y de las personas (Ovais 
\& Li, 2016; Waddock \& Steckler, 2016), lo que les permite confiar en su poder para transformar realidades inequitativas (Chell et al., 2016; Waddock \& Steckler, 2016). Esta dimensión tiene más carga en las creencias que en las prácticas, es decir, tiene un carácter cognitivo y afectivo asociado a una convicción sobre estados finales de la realidad que se consideran absolutamente cruciales para la persona (como la bondad, la justicia, el bienestar), lo que se explica desde el concepto específico de valores como estados metamotivacionales que trascienden situaciones específicas y son precursores de la acción (García-Villaverde \& Valencia, 2009). De acuerdo al análisis, se plantea la visión social como una dimensión fundamentalmente axiológica, que orienta la actividad emprendedora hacia el bien común como una misión de vida.

\section{Aprecio por prácticas sostenibles}

Esta dimensión da cuenta de experiencias que se articulan con las otras cuatro dimensiones del ES, por lo que resulta central para los participantes, quienes demuestran un estado de conciencia crítica que les posibilita saberse parte de un sistema interconectado (Nga \& Shamuganathan, 2010; Peredo et al., 2017) donde sus acciones tienen efectos en otros (Ovais \& Li, 2016). Este sentido de interconexión, de acuerdo con los participantes, parte de reconocerse a sí mismos como personas privilegiadas que tienen la responsabilidad de actuar.

Yo siempre sentía que uno es parte de la sociedad y no está solo, no es como que solamente haces lo que tú quieres. Todo está conectado (participante 5).

... yo creo que uno tiene la responsabilidad, si ha recibido muchas cosas, de devolver lo que ha recibido. Si yo he tenido la posibilidad de recibir una buena educación, tengo la responsabilidad de compartir (participante 2).

Si bien la literatura ejemplifica las formas en que se llevan a cabo prácticas sostenibles al emprender (Mair \& Martí, 2006; Zhang \& Swanson, 2014), no aclara las condiciones sobre las cuales se forma el sentido de sostenibilidad e interdependencia. En los participantes, las experiencias en contextos diversos nutren su sentido de interconexión que conlleva la conciencia sobre las consecuencias de las decisiones, buscando un equilibrio (Írengün \& Arikboga, 2015; Humbert \& Roomi, 2018; Whitman, 2011).

Mi papá se dedicaba a estudiar las variedades silvestres... Me llevaba a algunos de sus viajes. Entonces, yo he caminado por la sierra desde muy chiquito, tuve la oportunidad de aprender que somos parte de una misma naturaleza (participante 6).

Para estos ES, el Aprecio por prácticas sostenibles integra aspectos autobiográficos, afectivos, con un coherente razonamiento lógico que les permite procesar la información social de manera sensible y responsable, planificando soluciones puntuales y sostenibles a las problemáticas identificadas (Mair \& Martí, 2006).

Entonces, entiendes tus recursos, planificas y te proyectas, porque hay un tema a tener presente que es una suerte de equilibrio interno y organizacional... que la gente gane lo suficiente porque el trabajo amerita una remuneración, pero que sepan que la meta no es enriquecerse sino, sobre todo, que estén bien, que tengan un sentido y sepan que es posible actuar y que esto tiene un efecto y una duración en el tiempo (participante 7).

Esta dimensión representa una integración tanto de creencias como de prácticas, es decir, articula formas de pensar, sentir y leer el mundo, y luego ofrece posibilidades para ejecutar acciones concretas valiéndose de sus habilidades. Por esta razón, se propone esta dimensión como una variable actitudinal (Eagly \& Chaiken, 2005; Morris \& Maisto, 2011) que además es central en el comportamiento de los participantes porque predispone a los individuos a imaginar y evaluar positivamente distintas medidas de sostenibilidad y, adicionalmente, los impulsa a realizar prácticas sostenibles de acuerdo a las destrezas conductuales que hacen parte de las otras tres dimensiones que a continuación se detallan. 


\section{Capacidad innovadora}

Esta dimensión se basa en la necesidad de lograr un resultado original para garantizar la sostenibilidad y competitividad del emprendimiento (Sesen, 2013). Esto lleva a los participantes a generar soluciones innovadoras diversificando el talento como recurso que integra las habilidades de sus colaboradores.

Haces un poco de todo... las personas que son parte del equipo pueden hacer talleres, pueden reunirse con empresas, con organizaciones, elaborar un marco lógico, organizar eventos. En otras palabras, al final tienes que tener distintas llaves, ¿no? (participante 8).

En efecto, como señala la literatura, la innovación se desarrolla y nutre en el intercambio de capital intelectual y social (Sastre-Castillo et al., 2015), a lo que los participantes integran el uso de tecnologías de la información (TICS) y redes que potencializan el alcance y capacidad de sus proyectos.

Las redes sociales, su efecto multiplicador... hace poco hemos entrado a este proyecto con las TICS porque cada vez, como demanda educativa presencial, era más difícil atender y el ámbito de atención, entonces se está llegando a más capacidad con el uso de medios (Participante 13).

Por otro lado, en contraposición a la teoría que sostiene que la innovación conlleva a generar plataformas de recursos para la ejecución y planificación programada (Nga \& Shamuganathan, 2010; Pless, 2012), los participantes reportan hacer innovación de tipo emergente, es decir, atendiendo sobre la marcha las demandas del día a día. Esto se podría explicar por la poca consolidación de un ecosistema emprendedor social local, que dé sustento a la sistematización de innovaciones exitosas (Vera et al., 2016). En las experiencias de los entrevistados, la innovación emergente garantiza flexibilidad ante las demandas del entorno de manera sostenible.
No podíamos entrar con planes tan grandes pues había empresas clientes que tenían necesidades muy puntuales. Entonces, tuvimos que flexibilizarlos... porque, por lo menos, tenemos aunque sea un día para que vaya entrando el tema ambiental y que vayan entendiendo lo que necesitan hacer a largo plazo (participante 10).

Los entrevistados están conscientes de la necesidad de la innovación para encontrar nuevas formas de llegar a sus destinatarios, replanteando distintos aspectos del modelo de negocio con el fin de garantizar la viabilidad de su iniciativa social (Írengün \& Arikboga, 2015; Nga \& Shamuganathan, 2010). Se trata entonces de una dimensión eminentemente práctica que da cuenta del dominio de competencias, sumada con rasgos de apertura intelectual y a la experiencia (Caballero et al., 2014).

\section{Habilidad para desarrollar redes de contacto}

Esta dimensión basada en las relaciones personales que garantizan apoyo (Nga \& Shamuganathan, 2010) es considerada por los participantes como una condición fundamental del emprendimiento, el cual reconocen como una iniciativa colectiva, conjunta, inviable en solitario.

Esto es una construcción colectiva, o sea, yo he cumplido un rol, pero digamos que no sería explicable por la labor de una persona, sino los líderes, las otras personas tienen que estar ahí. Otros profesionales han sumado y han generado sinergias para que esto funcione (participante 9).

Entonces, el sano funcionamiento de la organización solo se hace posible pensando en colectivo, integrando diversas experiencias y conocimientos de manera respetuosa y difundiéndolos más allá de las fronteras geográficas e integrando capacidades y poniendo en valor aprendizajes conjuntos. 
Una de las características de la Asociación es que hay quienes han vivido la experiencia en carne propia, hay trabajadoras, estudiantes, profesionales, mujeres, varones, jóvenes, personas mayores, voluntarios, tanto peruanos como extranjeros. Es un equipo mixto en el que tratamos de ser lo más democráticos y abrirnos al mundo (participante 11).

Tener un proyecto de ciencia nos colocó en National Geographic, tener una asociación con una comunidad nativa nos ha dado una exposición más alláì de la que hubiera estado en capacidad cualquier otra empresa (participante 13).

Las redes de contacto se materializan en prácticas rigurosas de cuidado de los otros, lo que estaría en consonancia con el rasgo de consciencia y tesón como predictor de esta dimensión, según lo indica un estudio local (Caballero et al., 2014). Y, además, de acuerdo a la literatura general, se vincularía con habilidades de interacción como la extroversión para el apoyo, divulgación y éxito del proyecto, junto a la construcción de confianza, respeto, buena reputación basada en buenas relaciones y su oportuna divulgación (Alonso-Martínez, Gonzáles-Álvarez, \& Nieto, 2015; Gurrieri, 2013).

\section{Habilidad para generar retornos financieros}

Aunque los retornos financieros son claves para garantizar la sostenibilidad económica de la iniciativa social (Curry et al., 2016; İ́rengün \& Arikboga, 2015), esta es la dimensión más difícil de concretar para los participantes. La austeridad y recursividad resulta el primer mecanismo para solventar las limitaciones económicas iniciales del emprendimiento.

Entonces, al inicio tuì haces todo. ¿Quieìn hace el delivery? Nosotras. ¿Quieìn empaqueta el regalo? Tuì. Entonces, aprendes todo desde abajo... es un trabajo de poco a poco porque como emprendedores no es que tengas mucha plata (participante 16).

Una parte considerable de las empresas sociales de Lima no son totalmente autosostenibles y aún se dependen de donaciones (Vera et al., 2016) o fondos concursables y, dado que las ayudas internacionales para el país se han reducido en las últimas décadas, muchas iniciativas sociales están transitando hacia modelos de negocio para autofinanciarse (Farber et al., 2015):

Las donaciones que recibimos son bien puntuales, no es lo que nos sostiene. Lo que nos sostiene económicamente es la venta de los productos y los servicios... Somos una empresa, es importante velar por la rentabilidad de las actividades que hacemos... Se trata que sea sostenible dentro de la realidad en la cual trabajamos (participante 12).

En la empresa tenemos muchas donaciones, pero tenemos planeado varios modelos de negocio para hacer que empiece a caminar solo, ¿no? Por ejemplo, ahora vamos a plantear un pago retrospectivo cuando tengan un ingreso (participante 14).

Aunque los ES se pueden inspirar en modelos de creación de valor económico ya existentes (Curry et al., 2016; Rahdari et al., 2016), las finanzas parecen ser un factor complicado de garantizar en la práctica (Vera et al., 2016). Esta dimensión del ES en efecto se concreta en lo conductual, en la capacidad de hacer y, de acuerdo a un estudio local, se explica por rasgos de extroversión y de inestabilidad emocional, en tanto estos ES deben sobreponerse a la incertidumbre y ejecutar acciones que generen ingresos económicos en condiciones aún emergentes (Caballero et al., 2014; Vera et al., 2016).

\section{Interacción de las dimensiones del emprendimiento social}

Tras analizar las cinco dimensiones desde las experiencias transversales a todos los participantes, se plantea un modelo conceptual de las mismas, ubicándolas dentro de los constructos de creencias o prácticas y definiéndolas más específicamente como variables psicosociales que interactúan entre sí. La Figura 1 presenta las cinco dimensiones situadas dentro del modelo. 


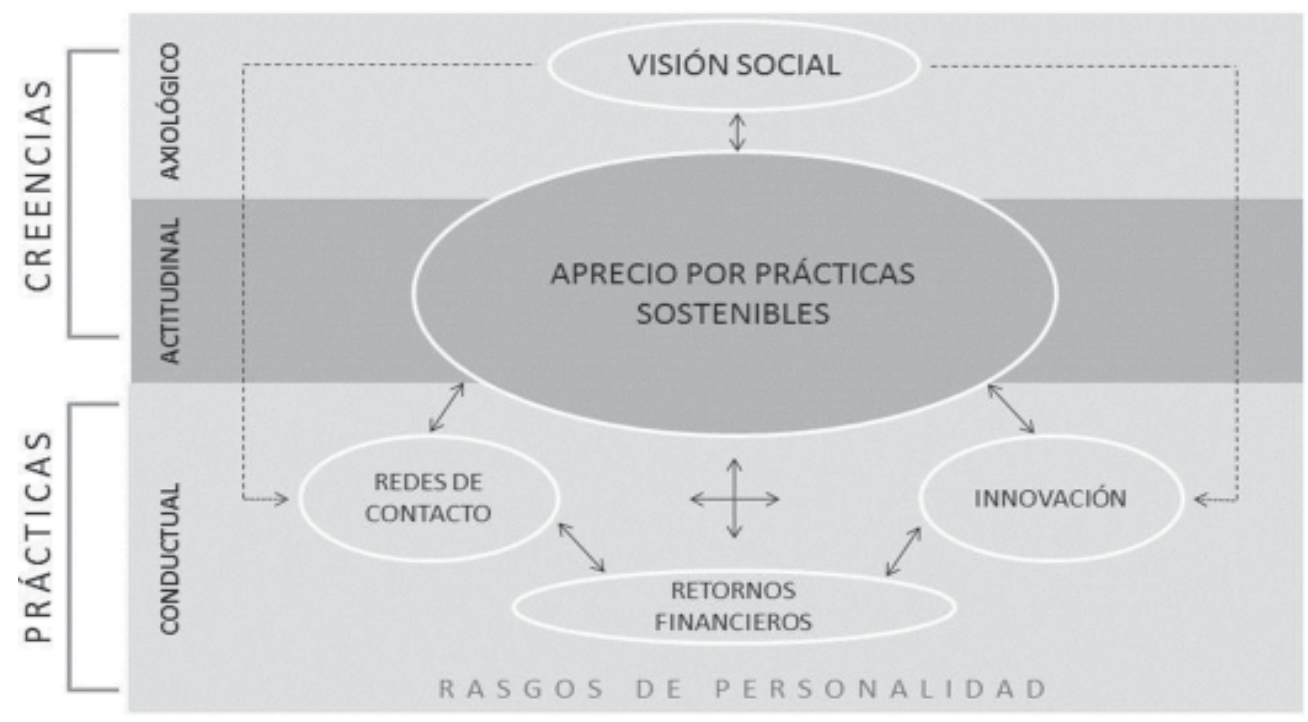

Figura 1. Modelo integrador de cinco dimensiones entendidas desde variables psicosociales.

La dimensión Aprecio por prácticas sostenibles (APS) ocupa un lugar central en las experiencias de los participantes. Esta dimensión se caracteriza por equilibrar cognición, afecto y conducta, situándose entre las creencias y las prácticas como una dimensión actitudinal, fundamental para la toma de decisiones y el desarrollo de acciones emprendedoras. El modelo plantea que el APS, en tanto juicio evaluativo sobre la sostenibilidad, concreta los elementos emotivos y axiológicos característicos de la visión social, ubicada en el plano más abstracto de los valores autotrascendentes para articularlos con conductas competentes y concretas representadas en las tres dimensiones situadas a nivel de prácticas: innovación, redes y retornos, las cuales dan soporte operativo al emprendimiento y además evidencian rasgos de personalidad de los participantes, que predecirían su propensión o facilidad a desarrollar dichos comportamientos.

El APS cumple el rol de dimensión central, articuladora y planificadora que integra las trayectorias de los participantes. La Figura 1 representa direcciones de relación entre todas las dimensiones, pues se trata de un modelo dinámico, no secuencial, sino simultáneo, en el que todos los componentes actúan al unísono, alineados a una toma de consciencia y actitud favorable hacia la sostenibilidad. Por lo que, aunque la teoría revisada sostiene que la visión social es la dimensión que funda la actividad emprendedora social (Írengün \& Arikboga, 2015; Ovais \& Li, 2016; Waddock \& Steckler, 2016), este estudio plantea un hallazgo novedoso al identificar, en los ES participantes, el APS como la dimensión central que los lleva a emprender socialmente y que los diferencia de personas que hacen actos caritativos o filantrópicos y de empresarios cuyo fin son las ganancias monetarias (Acs, Boardman \& McNeely, 2013; Sastre-Castillo et al., 2015). Estas personas, al igual que sus emprendimientos, suponen un hibrido entre lo social y lo comercial, donde la noción de sostenibilidad abandera su causa.

Es así que el APS, bajo la lógica de un pensamiento complejo de interdependencia, hace posible procesar información y experiencias socialmente sensibles (creencias) para articularlas con la ejecución de acciones que reequilibren el sistema (prácticas). El APS es la actitud distintiva que gatilla la orientación hacia el emprendimiento social. El modelo conceptual propuesto, con variables 
psicosociales claras y definidas para cada dimensión (valores, actitudes, conductas, rasgos), permite integrar y dar un estatus conceptual a cada una de estas dimensiones que, como ya se ha mencionado, son producto de abordajes disciplinarios diversos aún no plenamente articulados, el cual, al ser traducido a un lenguaje psicosocial común, facilitará el estudio, comprensión y posible formación-desarrollo de las mismas.

Por otra parte, el modelo permite caracterizar y organizar las interacciones entre las dimensiones de manera flexible según el contexto. Para el caso local específico, resulta pertinente seguir estudiando el APS como potenciador del fenómeno emprendedor social, siendo este, además, un resultado que cobra importancia en las actuales condiciones socioeconómicas del Perú: con un crecimiento emergente y acelerado, pero poco igualitario e inclusivo (Farber et al., 2015; Caballero et al., 2014) que requiere encontrar equilibrios y perspectivas de sustento futuros donde, por supuesto, el aprecio por nociones de sostenibilidad es totalmente pertinente.

\section{Conclusiones}

La investigación sobre las características personales y los procesos involucrados en el fenómeno del emprendimiento social debe ampliarse reconociendo los múltiples aspectos culturales implicados en el mismo para favorecer su desarrollo (Kliksberg, 2012). El análisis de las trayectorias de ES locales, según las cinco dimensiones, ha permitido identificar la relevancia de la dimensión actitudinal de APS como detonante del comportamiento emprendedor social en el contexto local peruano, lo cual es un aporte novedoso. En este sentido, posiciona el tema de la sostenibilidad no solo como un modo de ejecutar programas que garanticen la supervivencia del emprendimiento, sino como un posicionamiento psicológico, personal, complejo e integrador, que valora de manera sistémica e interdependiente las consecuencias de los propios actos y decisiones.
Este resultado, en un contexto local poco inclusivo, tiene mucho sentido para reflexionar sobre las posibilidades de llevar a cabo iniciativas que pueden considerarse al margen de la lógica convencional del emprendedor y resaltan las posiciones éticas y críticas que los emprendedores participantes asumen sobre su papel como posibles líderes responsables con la sociedad peruana. Entender con más detalle los procesos por los cuales se consolida el APS ofrecerá opciones para pensar en condiciones de formación de futuros ES. Por otra parte, entender las fortalezas y debilidades en todas las dimensiones posibilitaría generar una agenda de investigación y atención de necesidades puntuales de estos emprendimientos sociales para potenciar sus impactos y repercusiones positivas en la sociedad. Por ejemplo, el tema de retornos económicos sigue siendo difuso y requerirá de reflexiones más amplias sobre las condiciones que, en el ecosistema emprendedor social local (Vera et al., 2016), deben generarse para apoyar este requerimiento.

Se espera que los hallazgos del presente estudio sumen al conocimiento de un fenómeno emprendedor novedoso y socialmente comprometido y que en esa medida aporten a desencadenar un cambio de paradigma sobre las formas productivas convencionales; por lo tanto, se espera que el trabajo contribuya a orientar la educación, de los futuros líderes organizacionales, nutriéndola de actitudes compatibles con el desarrollo sostenible.

\section{Conflicto de intereses}

Los autores refieren que no existe ningún tipo de conflicto intereses.

\section{Responsabilidad ética}

Este estudio fue desarrollado por un equipo de investigadores interdisciplinario e interuniversitario en el marco de la supervisión y financiamiento del Consorcio de Universidades (Perú). Bajo el estricto rigor de esta entidad, el proyecto aseguró el 
cumplimiento de todos los estándares éticos exigidos por su comité, respecto al manejo de participantes e información. Se contó con los debidos protocolos, asegurando la voluntariedad y confidencialidad de la participación y se llevó a cabo un proceso de socialización de resultados dentro de los estándares exigidos por el Consorcio. Vale señalar también que en la presente investigación no se han llevado a cabo experimentos ni con seres humanos ni con animales.

\section{Contribución de autoría}

AVR: coordinación, concepción y diseño del estudio, recolección de datos, codificación y análisis de información, argumentación teórica, redacción principal del manuscrito y revisión final del documento.

MAPV: diseño y elaboración del instrumento, contacto con participantes, recolección de datos, argumentación teórica y revisión del documento.

AEP: desarrollo teórico de modelo conceptual, diseño y elaboración del instrumento, y revisión de discusión teórica final.

EFNL: revisión de literatura, construcción de argumentación teórica para la discusión de resultados y revisión de aspectos formales para presentación del manuscrito.

\section{Agradecimientos}

La financiación del presente estudio fue otorgada por el Consorcio de Universidades de Perú en el marco del Premio a la Investigación Interuniversitaria y Multidisciplinaria.

Agradecemos también el aporte del practicante de Psicología Social, Carlos Flores, quien contribuyó en dar el formato final al documento presentado.

\section{Referencias}

Abhi, S. (2017). Can Social Entrepreneurs do well by doing good? Blending social and economic value creation. An Investigation. Academy of Entrepreneurship Journal, 23(2), 1-21.

Acs, Z., Boardman, M., \& McNeely, C. (2013). The social value of productive entrepreneurship. Small Business Economics, 40(3), 785-796. doi: 10.1007/s11187-0119396-6

Alonso-Martínez, D., Gonzáles-Álvarez, N., \& Nieto, M. (2015). La innovación social como motor de creación de empresas. Universia Business Review. 1(47), 48-63. Recuperado de https://www.redalyc.org/articulo.oa ?id=433/43341001003

Austin, J., Stevenson, H., \& Wei-Skillern, J. (2006). Social and Commercial Entrepreneurship: Same, Different or Both? Entrepreneurship Theory and Practice, 30(1), 1-22. doi: 10.1111/j.1540-6520.2006.00107.x

Bacq, S., \& Alt, E. (2018). Feeling capable and valued: A prosocial perspective on the link between empathy and social entrepreneurial intentions. Journal of Business Venturing, 33(3), 333-350. doi: 10.1016/j.jbusven t.2018.01.004

Banco Mundial. (2016). Perú panorama general. Banco Mundial. Recuperado de http://www.bancomundial. org/es/country/peru/overview

Bikse, V., Rivza, B., \& Riemere, I. (2015). The Social Entrepreneur as a Promoter of Social Advancement. Procedia - Social and Behavioral Sciences, 185, 469478. doi: 10.1016/j.sbspro.2015.03.405

Brandstätter, H. (2011). Personality aspects of entrepreneurship: A look at five meta-analyses. Personality and Individual Differences, 51(3), 222-230. doi: 10.1016/j.paid.2010.07.007

Braun, V., \& Clarke, V. (2006). Using thematic analysis in psychology. Qualitative Research in Psychology, 3(2), 77-101. doi: 10.1191/1478088706qp063oa

Caballero, S., Fuchs, R. M., Prialé, M. A., \& Nga, K. H. (2014). The influence of the Big 5 personality traits on the social enterprise start-up intentions: A Peruvian case. Taylor's Business Review, 4(1), 87-107. 
Carter, J., Collins, L., \& Beal, B. (2017). Ethics, Faith, and Profit: Exploring the Motives of the U.S. Fair Trade Social Entrepreneurs. Journal of Business Ethics, 146(1), 185-201. doi: 10.1007/s10551-015-2934-4

Chell, E., Spence, L., Perrini, F., \& Harris, J. (2016). Social Entrepreneurship and Business Ethics: Does Social Equal Ethical? Journal of Business Ethics, 133(4), 619625. doi: 10.1007/s10551-014-2439-6

Choi, N., \& Majumdar, S. (2014). Social entrepreneurship as an essentially contested concept: Opening a new avenue for systematic future research. Journal of Business Venturing, 29(3), 363-376. doi: 10.1016/ j.jbusvent.2013.05.001

Consejo Nacional de Ciencia, Tecnología e Innovación Tecnológica [CONCYTEC]. (2016). Política Nacional para el Desarrollo de la Ciencia, Tecnología e Innovación Tecnológica - CTI. Lima, Perú. Recuperado de https://portal.concytec.gob.pe/index.php/publicacio nes/politica-nacional-de-cti

Creswell, J. (2013). Qualitative inquiry and research design. Choosing among five approaches. Londres: SAGE Publications.

Curry, J., Donker, H., \& Michel, P. (2016). Social entrepreneurship and indigenous people. Journal of Co-operative Organization and Management, 4(2), 108-115.

Defourny, J., \& Nyssens, M. (2017). Fundamentals for an International Typology of Social Enterprise Models. VOLUNTAS: International Journal of Voluntary and Nonprofit Organizations, 28(6), 2469-2497. doi: 10.1007/ s11266-017-9884-7

Eagly, A., \& Chaiken, S. (2005). Attitude research in the 21st century: The current state of knowledge. In D. Albarracín, B. T. Johnson, \& M. P. Zanna (Eds.), The Hanbook of Attitudes (pp. 743-767). Mahwah, New Jersey, USA: Lawrence Erlbaum Associates Publishers.

El Ebrashi, R. (2013). Social entrepreneurship theory and sustainable social impact. Social Responsibility Journal, 9(2), 188-209. doi: 10.1108/SRJ-07-2011-0013

Estrin, S., Mickiewicz, T., \& Stephan, U. (2016). Human capital in social and commercial entrepreneurship. Journal of Business Venturing, 31(4), 449-467.
Farber, V., Caballero, S., Prialé, M. A., \& Fuchs, R. M. (2015). Social Enterprises in Lima: Notions and Operating Models. Journal of Entrepreneurship and Innovation in Emerging Economies, 1(1), 56-78.

Freixa, E. (2003). ¿Qué es conducta? Revista Internacional de Psicología Clínica y de la Salud, 3(3), 595-613. Recuperado de https://www.redalyc.org/ articulo.oa?id=337/33730310

García-Villaverde, P. M., \& Valencia, P. (2009). Factores de éxito del proceso de puesta en marcha de la empresa: valores culturales, redes sociales y ayudas públicas. Investigaciones Europeas de Dirección y Economía de la Empresa, 15(3), 101-117. doi: 10.1016/S11352523(12)60103-8

Ghezzi, P., \& Gallardo, J. (2013). Qué se puede hacer con el Perú. Ideas para sostener el crecimiento en el largo plazo. Lima: Universidad del Pacífico y Fondo editorial de la Pontificia Universidad Católica del Perú.

Giraldo-Mejía, W., \& Vara-Horna, A. (2018). El emprendimiento social de base universitaria en Latinoamérica ¿Están las universidades haciendo lo suficiente? Lima: Universidad de San Martín de Porres.

Global Entrepreneurship Monitor [GEM] (2018). Global Entrepreneurship monitor 2018/2019 Global Report. Recuperado de http://www.gemconsortium.org/

Graddy-Reed, A. (2018). Do Hybrid Firms Out-Provide Traditional Business Structures? An Examination of Prosocial Behavior in North Carolina Firms. Nonprofit and Voluntary Sector Quarterly, 47(6), 1223-1248. doi: 10.1177/0899764018786468

Gurrieri, A. (2013). Networking Entrepreneurs. The Journal of Socio-Economics, 47, 193-204.

Humbert, A. L., \& Roomi, M. A. (2018). Prone to «care»?: Relating motivations to economic and social performance among women social entrepreneurs in Europe. Social Enterprise Journal. 4(3), 312-327. doi: 10.1108/sej-11-2017-0058

Írengün, O., \& Arikboga, S. (2015). The Effect of Personality Traits on Social Entrepreneurship Intentions: A Field Research. Procedia - Social and Behavioral Sciences, 195, 1186-1195. doi: 10.1016/j.sb spro.2015.06.172 
Kliksberg, B. (2012). Emprendedores sociales, los que hacen la diferencia. Buenos Aires: Grupo Editorial SRL.

Kristiansen, S., \& Ryen, A. (2002). Enacting their Business Environment: Asian Entrepreneurs in East Africa. African and Asian Studies, 1(3), 165-186. doi: 10.1163/ 156921002 X00040

Kuckertz, A., \& Wagner, M. (2010). The influence of sustainability orientation on entrepreneurial intentions - Investigating the role of business experience. Journal of Business Venturing, 25(5), 524-539. doi: 10.1016/ j.jbusvent.2009.09.001

Kunan \& Universidad Científica del Sur. (2019). Reporte sobre el estado del emprendimiento social y ambiental en el Perú. Lima: Fondo Editorial de la Universidad Científica del Sur. Recuperado de http:// www.reporteemprendimientosocial.pe/reporteemprendimiento-social.pdf

Latinobarómetro (2017). Informe 2017. Buenos Aires: Corporación Latinobarómetro. Recuperado de www.latinobarometro.org/LATDocs/F00006433InfLatinobarometro2017.pdf

Luca, M. (2017). Entrepreneurial Personality and Entrepreneurial Behaviour. Bulletin of the Transilvania University of $\mathrm{Bra}^{\circ} \mathrm{ov}$, Series VII: Social Sciences and Law, 10(1), 19-28.

Mair, J., \& Martí, I. (2006). Social entrepreneurship research: A source of explanation, prediction, and delight. Journal of World Business, 41(1), 36-44.

Mendoza-Abarca, K., \& Mellema, H. (2016). Aligning economic and social value creation through pay-whatyou-want pricing. Journal of Social Entrepreneurship, 7(1), 101-125. doi: 10.1080/19420676.2015.1015437

Morris, C., \& Maisto, A. (2011). Introducción a la Psicología (13 ed.). México: Pearson Prentice Hall.

Nga, J., \& Shamuganathan, G. (2010). The Influence of Personality Traits and Demographic Factors on Social Entrepreneurship Start Up Intentions. Journal of Business Ethics, 95(2), 259-282. doi: 10.1007/s10551009-0358-8

Ojeda, E., \& Rodríguez, A. (2015). La innovación en los emprendimientos sociales: una tipología. Debates IESA, 20(4), 26-30.
Ovais, M., \& Li, C. (2016). Personality Traits and their Effects on Social Entrepreneurship Intention. The International Journal of Business \& Management, 4(4), 222-226.

Peredo, A. M., Haugh, H. M., \& McLean, M. (2017). Common property: Uncommon forms of prosocial organizing. Journal of Business Venturing, 33(5), 591602. doi: 10.1016/j.jbusvent.2017.11.003

Pless, N. (2012). Social Entrepreneurship in Theory and Practice - An Introduction. Journal of Business Ethics, 111(3), 317-320. doi: 10.1007/s10551-012-1533-x

Pollack, J., Rutherford, M., Seers, A., Coy, A., \& Hanson, S. (2016). Exploring entrepreneurs' social network ties: Quantity versus quality. Journal of Business Venturing Insights, 6, 28-35. doi: 10.1016/j.jbvi.2016.09.001

Popoviciu, I., \& Popoviciu, S. (2011). Social entrepreneurship, social enterprise and the principles of a community of practice. Revista de Cercetare si Interventie Sociala, 33, 44-55.

Pralahad, C. (2010). The Fortune at the Bottom of the Pyramid: Eradicating Poverty through Profits ( $\left.5^{\mathrm{a}} \mathrm{ed}.\right)$. New Jersey: Pearson Prentice Hall.

Rahdari, A., Sepasi, S., \& Moradi, M. (2016). Achieving Sustainability through Schumpeterian Social Entrepreneurship: The Role of Social Enterprises. Journal of Cleaner Production, 137(20), 347-360.

Reckwitz, A. (2002). Toward a Theory of Social Practices. A Development in Culturalist Theorizing. European Journal of Social Theory, 5(2), 243-263.

Rodrigo, M. J., Rodríguez, A., \& Marrero, J. (1993). Las teorías implícitas: una aproximación al conocimiento cotidiano. Madrid: Visor.

Sastre-Castillo, M., Periz-Ortiz, M., \& Danvila-Del Valle, I. (2015). What Is Different about the Profile of the Social Entrepreneur? Nonprofit Management \& Leadership, 25(4), 349-369. doi: 10.1002/nml.21138

Savitz, A., \& Weber, K. (2006). The Triple Bottom Line: How today's Best-Run Companies Are Achieving Economic, Social, and Environmental Success. San Francisco: Jossey-Bass.

Schwartz, S. (1992). Universals in the content and structure of values: Theoretical advances and empirical tests in 20 countries. In M. P. Zanna (Ed.), Advances in 
experimental social psychology (pp. 1-65). San Diego: Academic Press.

Schwartz, S. H. (2010). Basic values: How they motivate and inhibit prosocial behavior. In M. Mikulincer, \& M. Shaver (Eds.), Prosocial motives, emotions, and behavior: The better angels of our nature (pp. 221241). Washington D.C.: American Psychological Association. doi: 10.1037/12061-012

Sengupta, S., Sahay, A., \& Croce, F. (2017). Conceptualizing social entrepreneurship in the context of emerging economies: an integrative review of past research from BRIICS. International Entrepreneurship and Management Journal, 14(4), 771-803. doi: 10.1007/ s11365-017-0483-2

Serrano, R. (2010). Pensamientos del profesor: un acercamiento a las creencias y concepciones sobre el proceso de enseñanza-aprendizaje en la Educación Superior. Revista de Educación, 352, 267-287.

Sesen, H. (2013). Personality or environment? A comprehensive study on the entrepreneurial intentions of university students. Education + Training, 55(7), 624-640. doi: 10.1108/ET-05-2012-0059

Shove, E., Pantzar, M., \& Watson, M. (2012). The dynamics of social practice: Everyday life and how it changes. London: SAGE.

Smith, C., Smith, J., \& Shaw, E. (2017). Embracing digital networks: Entrepreneurs' social capital online. Journal of Business Venturing, 32(1), 18-34. doi: 10.1016/ j.jbusvent.2016.10.003

Vera, A., Prialé, M. A., Fuchs, R. M., Espinosa, A., Seminario, M., \& Ninahuanca, E. F. (2016). Hacia una comprensión del ecosistema emprendedor social peruano: contexto y características del emprendimiento social en Lima. Ciências Sociais Unisinos, 52(3), 343-353.
Waddock, S., \& Steckler, E. (2016). Visionaries and Wayfinders: Deliberate and Emergent Pathways to Vision in Social Entrepreneurship. Journal of Business Ethics, 133(4), 719-734. doi: 10.1007/s10551-014-2451-x

Weerawardena, J., \& Sullivan, G. (2006). Investigating social entrepreneurship: A multidimensional model. Journal of World Business, 41(1), 21-35. doi: 10.1016/ j.jwb.2005.09.001

Whitman, J. (2011). Social entrepreneurship: An overview. In W. Bygrave, \& A. Zacharakis (Eds.), Entrepreneurship (pp. 563-595). New York: John Wiley \& Sons.

Willig, C. (2013). Introducing Qualitative Research in Psychology. New York: McGraw Hill Education.

Young, R. (2006). For what it is worth: Social value and the future of social entrepreneurship. In A. Nicholls (Ed.), Social Entrepreneurship: New Models of Sustainable Social Change (pp. 56-73). New York: Oxford University Press.

Zhang, D., \& Swanson, L. (2014). Linking Social Entrepreneurship and Sustainability. Journal of Social Entrepreneurship, 5(2), 175-191. doi: 10.1080/1942 0676.2014.880503

Zhao, H., \& Seibert, S. (2006). The Big Five personality dimensions and entrepreneurial status: A metaanalytical review. Journal of Applied Psychology, 91(2), 259-271. doi: 10.1037/0021-9010.91.2.259

Zozimo, R., Jack, S., \& Hamilton, E. (2017). Entrepreneurial learning from observing role models. Entrepreneurship \& Regional Development, 29(9-10), 889-911. doi: 10.10 80/08985626.2017.1376518 


\section{Ángela Vera Ruiz}

Pontificia Universidad Católica del Perú, Perú.

Candidata a Doctora en Psicología por la Pontificia Universidad Católica del Perú. Magister en Estudios Culturales. Investiga temas de emprendimiento, emprendimiento social, comportamiento y cultura organizacional en un marco crítico de estudios laborales.

ORCID: http://orcid.org/0000-0002-8491-3979

Autora corresponsal: averar@pucp.pe

María Ángela Prialé Valle

Universidad del Pacífico, Perú.

Magíster en Desarrollo y Ayuda Internacional por la Universidad Complutense de Madrid. Investiga temas de Responsabilidad Social Empresarial, sostenibilidad empresarial, negocios inclusivos y emprendimiento social.

ORCID: http://orcid.org/0000-0003-4914-6388

priale_ma@up.edu.pe

Agustín Espinosa Pezzia

Pontificia Universidad Católica del Perú, Perú.

Doctor en Psicología Social por la Universidad del País Vasco (España). Investiga temas de psicología social y psicología política vinculados a procesos de construcción de identidades colectivas y relaciones intergrupales.

ORCID: http://orcid.org/0000-0002-2275-5792

agustin.espinosa@pucp.pe

Erle Frances Ninahuanca López.

Universidad del Pacífico, Perú.

Bachiller en Psicología por la Pontificia Universidad Católica del Perú. Cuenta con experiencia en investigación de diversos temas de Gestión Humana (desarrollo del talento, clima laboral, reclutamiento y selección).

ORCID: http://orcid.org/0000-0001-8980-8708

ef.ninahuancal@up.edu.pe 